\title{
Soft Wireless Battery-free UHF RFID Stretchable Sensor based on Microfluidic Technology
}

DOI:

10.1109/JRFID.2019.2912959

\section{Document Version}

Accepted author manuscript

Link to publication record in Manchester Research Explorer

\section{Citation for published version (APA):}

Pan, K., Teng, L., Ting, L., Zhou, X., Stokes, A. A., \& Hu, Z. (2019). Soft Wireless Battery-free UHF RFID Stretchable Sensor based on Microfluidic Technology. IEEE Journal of Radio Frequency Identification. https://doi.org/10.1109/JRFID.2019.2912959

\section{Published in:}

IEEE Journal of Radio Frequency Identification

\section{Citing this paper}

Please note that where the full-text provided on Manchester Research Explorer is the Author Accepted Manuscript or Proof version this may differ from the final Published version. If citing, it is advised that you check and use the publisher's definitive version.

\section{General rights}

Copyright and moral rights for the publications made accessible in the Research Explorer are retained by the authors and/or other copyright owners and it is a condition of accessing publications that users recognise and abide by the legal requirements associated with these rights.

\section{Takedown policy}

If you believe that this document breaches copyright please refer to the University of Manchester's Takedown Procedures [http://man.ac.uk/04Y6Bo] or contact uml.scholarlycommunications@manchester.ac.uk providing relevant details, so we can investigate your claim.

\section{OPEN ACCESS}




\title{
Soft Wireless Battery-free UHF RFID Stretchable Sensor based on Microfluidic Technology
}

\author{
Kewen Pan, Lijun Teng, Leng Ting, Zhou Xinyao, Adam A. Stokes and Zhirun Hu
}

\begin{abstract}
Stretchable microfluidic sensors have recently been developed and employed in many sensing applications. However, they are still in their early stage of development and facing considerable challenges, such as practical system integration due to the limitation of costs, wired connections and sensitivity. In this work, we present a compact soft wireless battery-free RFID stretchable sensor based on microfluidic technology. The proposed RFID sensor has high sensitivity and is able to be stretched to $160 \%$ of its original size. Moreover, the sensor is completely wireless powered and battery-free. It can be fully embedded into sensor carriers such as robots to eliminate wires, reduce the weight and increase the agility. Difference to known received signal strength indicator (RSSI) techniques for RFID sensing monitoring, resonance frequency shift is used in this work, significantly increasing the measurement accuracy, usefulness and reliability for many practical applications.
\end{abstract}

Index Terms -Ecoflex, microfluidic antenna, stretchable sensor, metamaterilas, radio frequency identification (RFID).

\section{INTRODUCTION}

Passive RFID technology in UHF band (860MHz-960MHz) utilizes RF signals to detect and identify specific objects [1]. Nowadays there is a growing interest in exploring the use of RFID technologies in sensing and monitoring [2]. Stretch monitoring using passive RFID technology was first demonstrated in [3] and further developed in [4], [5]. However, the deformable metal wire RFID tags in [5] have very limited lifetime and poor repeatability due to the fatigue of metal wire. E-textile works in [4] overcome the metal fatigue problem by using stretchable conductive polymers. However, the measurement depends on the received signal strength indicator (RSSI) which is not only affected by the antenna gain, but also varies with distance between the RFID tag and the reader antenna. Therefore, an additional reference tag located in a specific position is required to calibrate the distance effect. Furthermore, coupling and polarization effects between sensor and the reference tag have to be considered because they are not constant, especially when tags are bended or twisted. ${ }^{1}$

K. Pan, T. Leng, X. Zhou and Z. Hu are with the School of Electrical and Electronic Engineering, University of Manchester, Manchester M13 9PL, U.K. (Corresponding author: Z. Hu and e-mail: z.hu@manchester.ac.uk).

L.Teng and A. Stokes are with the School of Engineering, Institute for Integrated Micro and Nano Systems, The University of Edinburgh, The King's Buildings, Edinburgh, United Kingdom.

This is a joint article and these authors contributed equally to this work.
Benefiting from the development of microfluidic technology and the discovery of non-toxic, room-temperature liquid alloys made with gallium, indium (eGaIn) and tin (Galinstan), researchers have successfully fabricated liquid conductors and antennas without metal fatigue problem [22], [23]. Impressive works on deformable soft antennas, such as stretchable microfluidic antennas made with liquid metal and hybrid silicone [8], microfluidic serpentine antennas with reversible stretchability and mechanical frequency modulation ability [9] have been demonstrated. As the fabrication process becomes mature, mechanically tunable soft antenna was first reported using soft lithographic and rapid prototyping methods in [10]. After that, meandered dipole based stretchable RFID sensor has been reported in [11] and [12], where the measurement of stretch is based on RSSI and reference tag is still be required. In [13], a wireless stretchable RFID sensor which successfully converted the tensile elongation to frequency shift rather than signal strength was reported, significantly advanced the measurement reliability because the distance and angle between the tag and reader antennas are no longer relevant to the measurement accuracy. However, the antenna in [13] is a foot away from practical applications because of its lower Q factor, less sensitivity and nonlinearity.

In this work, the antenna structure is evolved from metamaterial inspired split ring resonator (SRR), operating at its second electric resonant mode [14], which overcomes the disadvantages of meandered dipoles as stretching sensing antennas in [11-13]. A T-matching network [15] was connected for conjugate matching with the RFID chip (Impinj Monza R6). The antenna has the size of $3.3 \times 3.5 \times 0.23 \mathrm{~cm}^{3}$ and the detection range of $6.7 \mathrm{~m}$ with the standard $3.28 \mathrm{~W}$ Equivalent Isotropic Radiated Power (EIRP).

Antenna design, fabrication process and measurement results will be presented in Section II, III and IV respectively, followed by discussions on the tests of antenna bending and rolling effects. Finally, a soft actuator monitoring system will be demonstrated.

\section{Design of Metamaterial Inspired LiQuid Metal Soft ANTENNA}

SRR is one of the most commonly used metamaterial structures and was first proposed in 1999 [16]. The strong coupling effects between the rings make the structure very sensitive to the change of the ring length and gap width. Such 
(a)

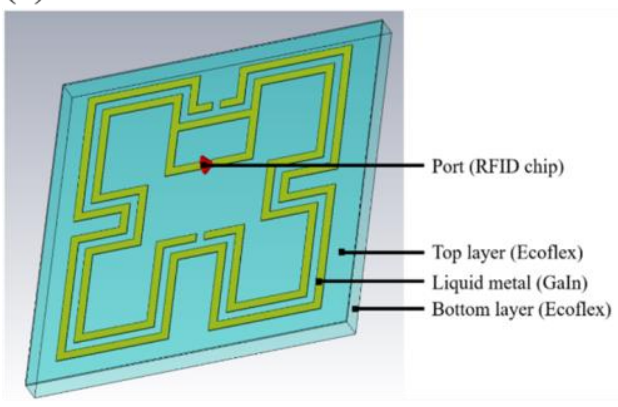

(b)

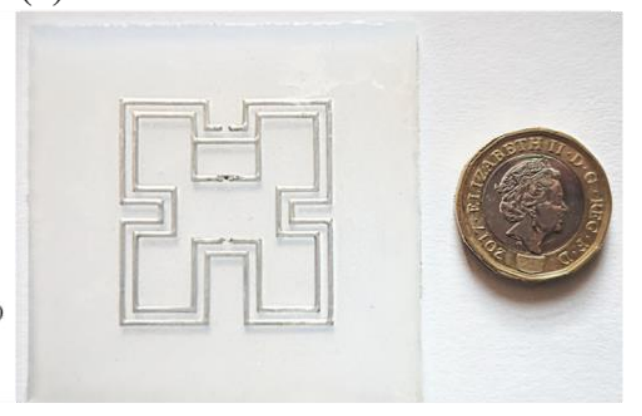

(c)

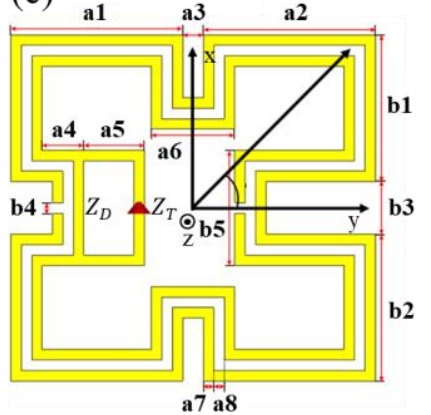

Fig. 1. SRR RFID stretchable sensor. (a) Antenna structure (red corn is feed port). (b) Fabricated device (3.3cm $\times 3.5 \mathrm{~cm})$. (c) Geometrical parameters of the antenna.

characteristics provide higher sensitivity compared with those of the meandered line antennas [5]. A SRR inspired antenna can have multimode resonances. Its fundamental resonance mode has been proved having low radiation efficiency [14], [17][19]because the radii of the rings are much smaller than the corresponding free space wavelength, which leads to low radiation resistance and efficiency [18]-[20]. Therefore, second resonance mode was chosen in this work. The antenna consisted of an SRR radiator, T-matching network and a RFID chip, as shown in Fig. 1(a) and (b). The antenna and matching network were designed and simulated using commercial EM simulation software CST MWS [26]. In the simulation, the liquid alloy was modelled as metal with a conductivity of $2.3 \times$ $10^{6} \mathrm{~S} / \mathrm{m}$ [20]. The geometrical parameters of the antenna are illustrated in Fig. 1(c): $a_{1}=a_{2}=16.5 \mathrm{~mm}, a_{3}=2 \mathrm{~mm}, a_{4}=4 \mathrm{~mm}$, $\mathrm{a}_{5}=5.8 \mathrm{~mm}, \mathrm{a}_{6}=8 \mathrm{~mm}, \mathrm{a}_{7}=\mathrm{a}_{8}=1 \mathrm{~mm}, \mathrm{~b}_{1}=\mathrm{b}_{2}=14 \mathrm{~mm}, \mathrm{~b}_{3}=5 \mathrm{~mm}$, $\mathrm{b}_{4}=1 \mathrm{~mm}, \mathrm{~b}_{5}=11 \mathrm{~mm}$. The thickness of bottom layer is $2.3 \mathrm{~mm}$ with $0.1 \mathrm{~mm}$ thick liquid channel. The upper layer is $0.2 \mathrm{~mm}$ thick for sealing purpose. The second resonance mode of the SRR antenna has been studied [21]. The radiation resistance in this resonance mode is approximately 7 times higher than that of the same half wave length dipole. The input impedance of the commercial RFID chip (Impinj, Monza R6) is 12-j120 $\Omega$ at $915 \mathrm{MHz}$. The T-matching network, which connects the chip to the inner ring of the SRR antenna, is required to provide a conjugate matching between the chip and antenna for maximum power efficiency. The input impedance of the antenna is [15]:

$$
Z_{\text {in }}=\frac{2 Z_{t} n^{2} Z_{a}}{2 Z_{t}+n^{2} Z_{a}}
$$

where $Z_{a}$ is the input impedance of the SRR antenna itself, $Z_{t}$ is the input impedance of T-matching network, $n$ is current ratio between two arms. The overall input impedance $Z_{\text {in }}$ can therefore be designed for conjugate matching with the RFID chip.

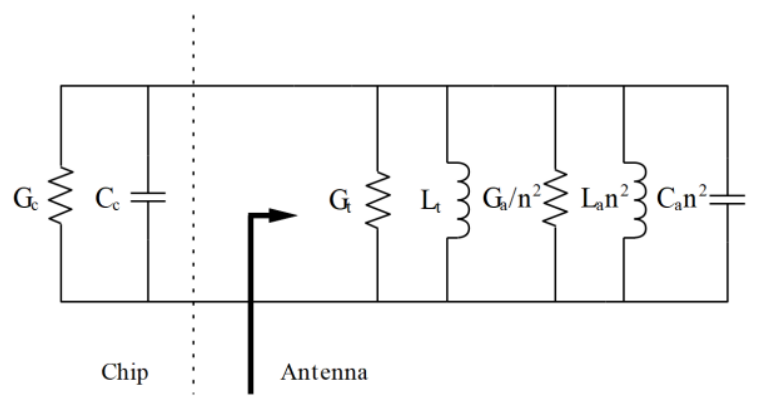

Fig. 2. Antenna equivalent circuit model (Left is RFID chip side and right is antenna side).

In the second resonance mode of SRR structure, the surface current distributions in both rings are of opposite directions and phase [14]. The resonance condition can be analysed using equivalent circuit model [19], as shown in Fig.2. $G_{c}, G_{t}$, and $G_{n}$ are the conductance of RFID chip, T-matching network loss and SRR antenna (caused by loss and radiation) respectively. $C_{c}$ and $C_{a}$ represent the RFID chip input capacitor and SSR antenna parasitic capacitor where $C_{a}=\pi L_{x} C_{p u l}[19]\left(L_{x}\right.$ is the side length of antenna, $C_{p u l}$ is per unit length capacitor). $L_{t}$ and $L_{a}$ are the T-matching shorter arm equivalent inductor and antenna inductor. Resonance frequency $f_{0}$ can be expressed as:

$$
f_{0}=\frac{1}{2 \pi \sqrt{L_{\text {total }} \text { total }}}
$$

where $L_{\text {total }}=\frac{L_{t} L_{a} n^{2}}{L_{a} n^{2}-L_{t}}$ and $C_{\text {total }}=C_{c}+C_{a} n^{2}$. It can be seen that the resonance frequency decreases significantly due to the transformation of T-matching network and RFID chip capacitor. Therefore, both T-matching network and SRR antenna behave inductively. Under stretched condition, $L_{t}$ and $L_{a}$ increase because of longer current path, resulting the resonance frequency move down to lower frequencies. The measurement results between different stretching levels and frequency shifts are discussed in the following sections.

\section{SOFT ANTENNA FABRICATION}

Galinstan is a generic name of a series of room-temperature eutectic liquid alloys which consist of gallium (Ga), indium (In), and tin (Sn). Galinstan has desirable properties such as low toxicity and low reactivity of its component metals. Therefore, 
Galinstan is an excellent substitution for some conventional conductive liquid, such as toxic mercury and reactive sodium-potassium alloy. Galinstan used in this work has a typical composition of $68.5 \% \mathrm{Ga}, 21.5 \% \mathrm{In}$, and $10 \%$ Sn with the electrical conductivity of $2.3 \times 10^{6} \mathrm{~S} / \mathrm{m}$. It also has a large surface tension and great wetting properties on the surface of Ecoflex or other silicone rubber materials. Galinstan, therefore, can afford stable and reliable connections in the elastic channels [10] as it tends to hold their position even when a device is twisted, stretched, or severely folded.

Ecoflex is one of the most commonly used substrate materials in fluidic devices due to its attractive high elasticity, uncomplicated fabrication, easy casting properties and excellent biocompatibility. In this work, we used Ecoflex type 00-50 to make elastic substrate of the SRR antenna. The elongation at break of Ecoflex 00-50 in tensile strength test with ASTM D412 system is $980 \%$ (defined as the percentage change in length or $\left(\frac{l-l_{0}}{l_{0}}\right) \times 100 \%$, [22]).

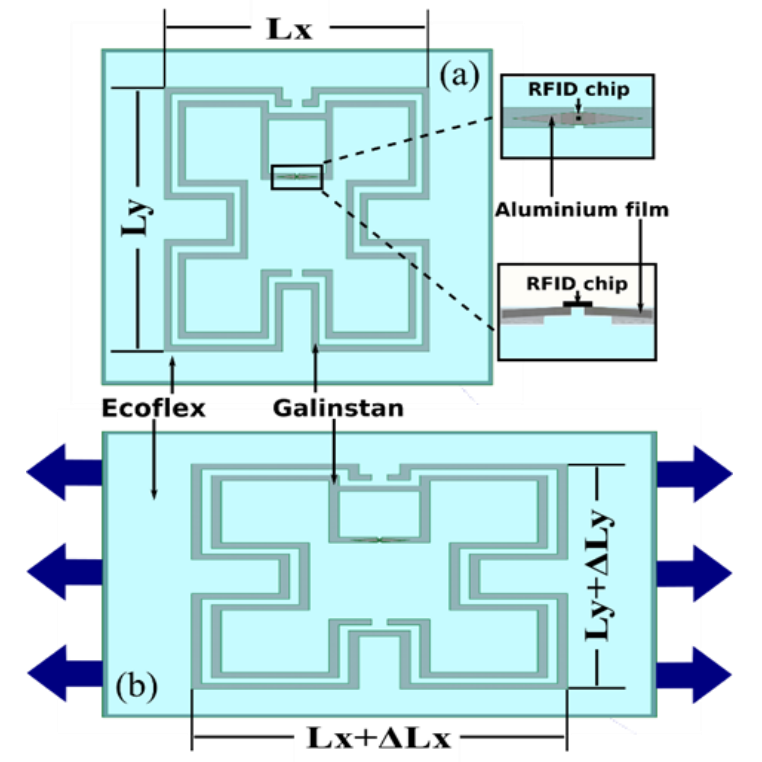

Fig. 3. (a) Top view of the Ecoflex liquid metal SRR antenna with detailed cross-section drawing of the RFID chip insertion. The initial length, width and thickness of the antenna are $\mathrm{L}_{\mathrm{x}}=33 \mathrm{~mm}, \mathrm{~L}_{\mathrm{y}}=35 \mathrm{~mm}$ and $\mathrm{L}_{\mathrm{z}}=2.5 \mathrm{~mm}$ respectively. The channels with filled Galinstan in the Ecoflex substrate are $1 \mathrm{~mm}$ in the width. The RFID chip was bonded on an aluminum film then inserted in the fluidic channels to connect with Galinstan; (b) when the antenna is under stretching, the length in $\mathrm{X}$ direction, the width in $\mathrm{Y}$ direction and the thickness in $\mathrm{Z}$ direction become $\mathrm{L}_{\mathrm{x}}+\Delta \mathrm{L}_{\mathrm{x}}, \mathrm{L}_{\mathrm{y}}+\Delta \mathrm{L}_{\mathrm{y}}$ and $\mathrm{L}_{\mathrm{z}}+\Delta \mathrm{L}_{\mathrm{z}}$ respectively.

Fig. 3(a) and (b) show the structure of the antenna without and with stretching respectively. Deformation of the antenna under stretch is significantly influenced by Poisson's effect. For example, when the antenna is stretched in one direction, it becomes narrower and thinner in other directions. Such phenomenon changes the thickness of the antenna substrate and also affects its resonance frequency. The Poisson's ratio $(v)$ of Ecoflex $00-50$ is 0.49 which means this material is a nearly incompressible material which deforms elastically even under a very small strain. In Fig. 3(a), the original length, width and thickness of the antenna are represented by $L_{x}, L_{y}$ and $L_{z}$ respectively. When the antenna is stretched in $\mathrm{X}$ direction $\left(L_{x}+\right.$ $\Delta L_{x}$ ), as shown in Fig. 3(b), the thickness and width change to
$L_{y}+\Delta L_{y}$ and $L_{z}+\Delta L_{z}$. Eq. (3) shows the relationship between $\Delta L_{y} / L_{y}, \Delta L_{z} / L_{z}$ and $L_{x}+\Delta L_{x}[22]:$

$$
\frac{\Delta L_{y}}{L_{y}}=\frac{\Delta L_{z}}{L_{z}}=\left(1+\frac{\Delta L_{x}}{L_{x}}\right)^{-v}-1
$$

It should be noticed when the length varies in $\mathrm{X}$ direction $\left(L_{x}\right)$, not only the width in Y direction, but the thickness will also change simultaneously.
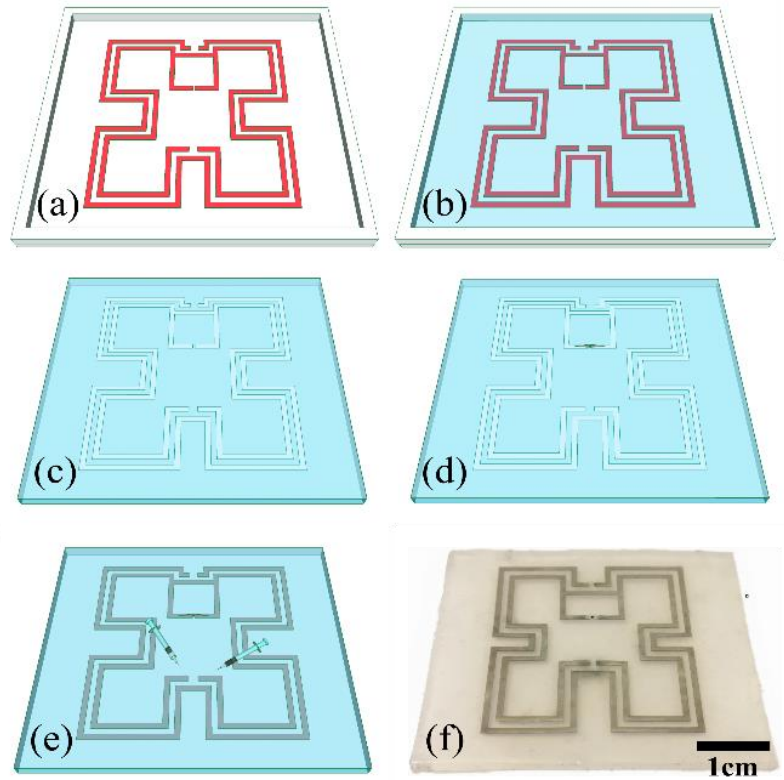

Fig. 4. Schematic illustration of the fabrication process and photograph of the stretchable fluidic antenna; (a) the soft-lithography mold made with $0.15 \mathrm{~mm}$ vinyl film (red) and $2 \mathrm{~mm}$ Acrylic substrate and frame (white); (b) uncured Ecoflex with height of $2 \mathrm{~mm}$ was poured into the soft-lithography mold; (c) the casted piece of Ecoflex was sealed with another $0.2 \mathrm{~mm}$ Ecoflex film; (d) the RFID chip bonded on an aluminum film was inserted into the channels in Ecoflex substrate; (e) two needles were used to inject Galinstan into the channels. (f) Photograph of the final prototype.

Fig. 4(a)-(e) illustrate the fabrication process of the stretchable sensor: (i) A soft-lithography mold to cast uncured Ecoflex with a $2 \mathrm{~mm}$ Acrylic board (Acrylic Cast, AMARI) and a piece of $0.15 \mathrm{~mm}$ self-adhesive vinyl film (CRAFTRKZO, d-c-fix $\left({ }^{\circledR}\right)$ were prepared. The Acrylic material, which was shaped with a laser cutter (Epilog Fusion), was used as the substrate and the frame in the mold. The vinyl film was used to form the structure of the channels in the mold. The profile of the antenna structure was cut on the vinyl film with a laser micro-machining system (Protolaser U3, LPKF) which contains an error rate less than 5\%. (ii) Part $\mathrm{A}$ and part $\mathrm{B}$ of Ecoflex (type 00-50, Reynolds Advanced materials) were mixed at the ratio of 1:1. The uncured Ecoflex with a controlled height of $2 \mathrm{~mm}$ was degassed under vacuum for five minutes and then poured into the soft-lithography mold. In order to fasten the curing, the mold was put into a convection oven at $65^{\circ} \mathrm{C}$ for 20 mins. (iii) The channels were sealed in the casted 2 $\mathrm{mm}$ Ecoflex layer with a piece of $0.2 \mathrm{~mm}$ Ecoflex film made with a spin coater. (iv) We made small incisions in the center of the thin Ecoflex layer to embed the RFID chip; the RFID chip (Monza R6; Impinj) was bonded on an Aluminum film with a bonding machine (FB-300 manual RFID wrapping machine) and inserted in the channels. (v) Two $0.3 \mathrm{~mm}$-diameter needles 
were used to inject Galinstan into the channels through positive pressure and remove air. (vi) In the end, a very thin film of uncured Ecoflex was coated on the surface of this antenna to seal all the holes.

\section{ANTENNA MEASUREMENT}

Voyantic Tagformance measurement system was used for the RFID tag measurement as shown in Fig. 5. The RFID reader contains a signal generator, transmitter, power detector and receiver. The RFID antenna receives the signal and power from the reader and activates its chip which in turn modulates and backscatters the signal to the reader antenna.

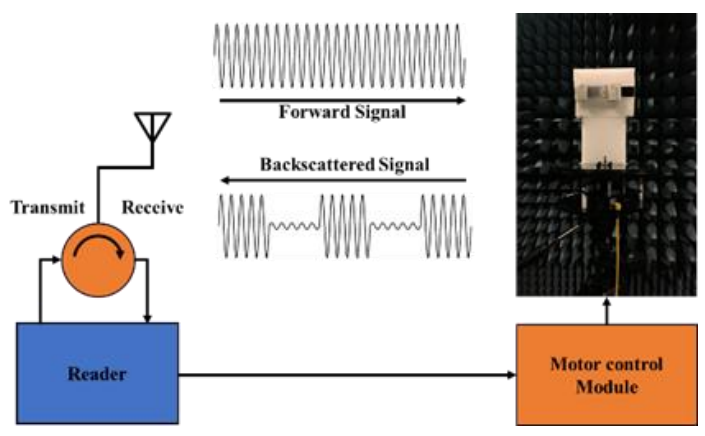

Fig. 5. RFID measurement set up with Voyantic Tagformance.

The minimum power that the RFID tag requires can be expressed as:

$$
P_{t a g}=\frac{P_{t h}}{\tau G_{t a g}}=\left[P_{t}-L_{c a b l e}+G_{t}-F S P L\right]_{d B}
$$

where $P_{t}, G_{t}$ are the minimum transmitted power and gain of the reader antenna, $L_{\text {cable }}$ and FSPL represent cable loss and free space loss which can be calibrated by using a standard tag before measurement. $P_{t h}$ is the minimum threshold of the power required to activate the RFID chip, $G_{t a g}$ is the gain of the tag antenna. $\tau$ is the matching factor, which describes the conjugate impedance matching between the antenna and RFID chip.

$P_{t h}$ and $\tau$ are constant for a specific frequency, the minimum power that can power on the chip is inverse proportional to the antenna gain $G_{t a g}$, which can be derived from measured $P_{t}$ :

$$
G_{\text {tag }}=\left[P_{t h}-P_{t}+L_{\text {cable }}-G_{t}+F S P L\right]_{d B}
$$

Considering the detection distance requirements in real applications, theoretical read range $R_{\max }$ can be calculated for given EIRP as:

$$
R_{\text {max }}=\sqrt{\frac{P_{\max , E I R P}}{P_{\text {tag }}}} \cdot \frac{c}{4 \pi f}
$$

where $P_{\max , E I R P}=3.28 \mathrm{~W}, c$ is light speed and $f$ is the corresponded frequency.

\section{RESUlTS AND DiscUSSIONS}

The simulated antenna impedance variation with stretching is shown in Fig. 6(a). It can be seen that the peak values of both real and imaginary parts of the impedance shift to lower frequency when increasing the stretching, resulting in the decrease of resonance frequency.

The RFID antenna performance was measured in anechoic chamber. To measure the gain and resonance frequency of the antenna, the reader sweeps linearly from $800 \mathrm{MHz}$ to $1 \mathrm{GHz}$ with a $3 \mathrm{MHz}$ step. The theoretical reading range is calculated using Eq. (6) and plotted in Fig. 6(b). It can be found that the resonance frequency without stretching is $938 \mathrm{MHz}$ with $6.7 \mathrm{~m}$ read range and it drops to $818 \mathrm{MHz}$ at $60 \%$ strain. Therefore, the sensitivity is $5.71 \mathrm{MHz} / \mathrm{mm}$, doubled the previous result [13] $(2.65 \mathrm{MHz} / \mathrm{mm})$. When the antenna is stretched, its resonance frequency decreases whereas the read range increases due to longer electric length of the antenna and higher gain.

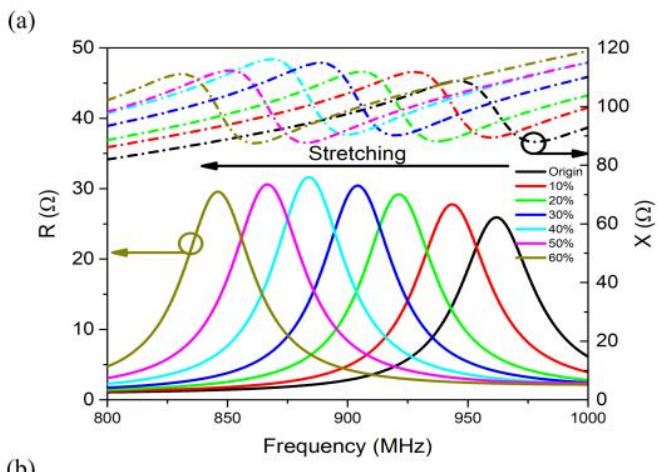

(b)

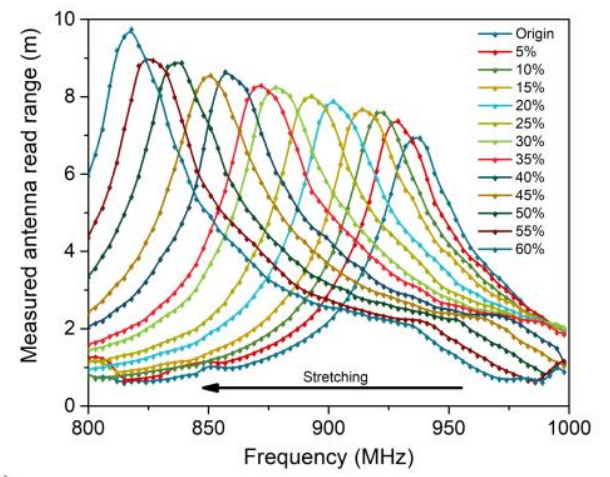

(c)

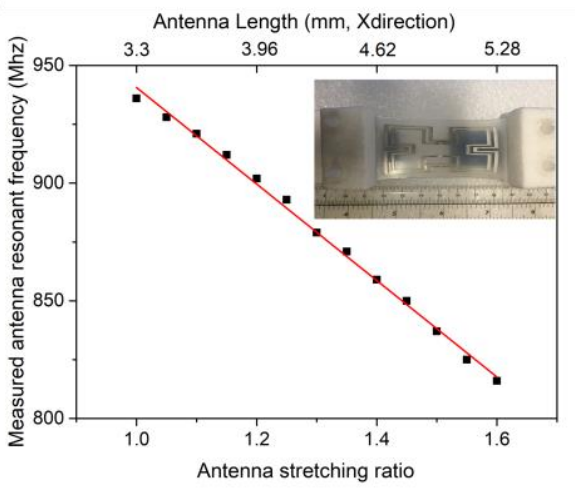

Fig. 6. (a) Simulated antenna impedance under different stretch conditions. (b) Measurement results of RFID tag read range and resonance frequency under different stretching conditions. (c) Antenna resonance frequency verses antenna stretching ratio (stretched length/ original length), the red line is linear fits of scatters. 
Fig. 6(c) illustrates the relationship between the resonance frequency and antenna stretching ratio. Two holders, which consist of low permittivity and low loss foam (71 IG-F, MK), were used to hold and stretch the antenna in Y-axis (inset Fig. 6(c)). The distance between the RFID sensor antenna and reader antenna is $80 \mathrm{~cm}$. To eliminate the influence of foam holders, calibration was performed every time when antenna length was changed. High stretchability up to $160 \%$ of the antenna length was tested with $5 \%$ per step. The antenna operates properly when being stretched to $160 \%$ and could be further stretched, yet, its resonance frequency will run out of the lowest allocated RFID frequency spectrum.

From Fig. 6(c), linear relationship between the resonance frequency and stretching length can be observed. Fitted formula of the relationship can be expressed as:

$$
f_{r}=-205 \times r+1145.6
$$

Eq. (7) can convert the stretching ratio $r$ directly from the measured resonance frequency. However, it should be noticed that two sides of the antenna were clamped by foam holders and the width is fixed. The ratio of stretching at the edge would be different from the central part. The same problem would happen in real applications, which affects antenna's resonance frequency. Therefore, it is necessary to calibrate the frequency-stretching ratio relationship after installing the antenna.
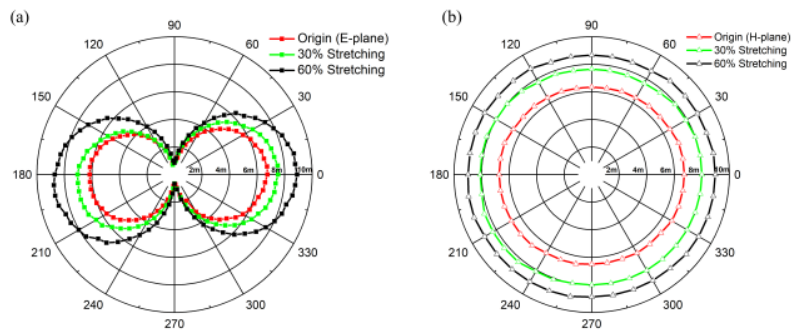

Fig. 7. Measured RFID antenna radiation pattern under different stretching length in E-plane (a) and H-plane (b) $(0 \%, 30 \%$ and $60 \%)$.

The radiation patterns of the RFID antenna under different stretching conditions were measured in both E-plane and H-plane (Antenna Measurement Studio 5.5, Diamond Engineering). For more intuitionistic demonstration, the read range was plotted instead of antenna gain. The data were recorded every 5 degrees rotation, as shown in Fig. 7. The measured radiation pattern is similar with dipole antenna which means the antenna is omnidirectional at H-plane and weakly directional at $\mathrm{E}$ plane. The maximum read range is approximately $6.7 \mathrm{~m}, 7.4 \mathrm{~m}$ and $9 \mathrm{~m}$ with $0 \%, 30 \%$ and $60 \%$ stretching, respectively. Except the read range, there is no significant distortion of radiation pattern under stretching.

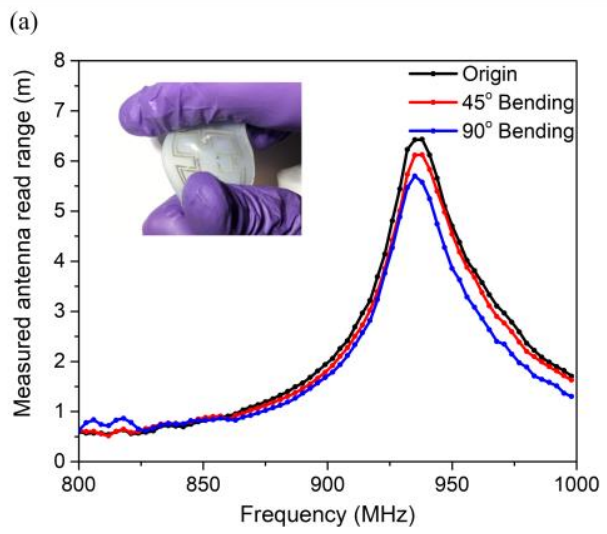

(b)

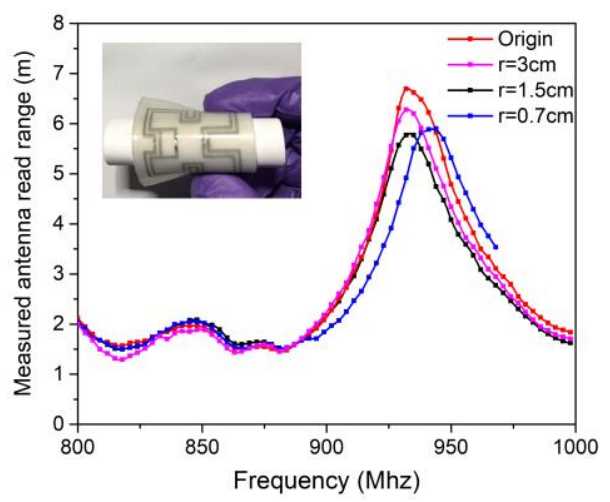

Fig. 8. (a) Antenna resonance frequency variation with different bending angle $\left(0^{\circ}, 45^{\circ}, 90^{\circ}\right)$. (b) Antenna resonance variation under rolling $(r=0.7,1.5,3, \infty)$.

Having demonstrated the relationship between stretching length and resonance frequency of the RFID stretch sensor, we go further to investigate the effects of bending and rolling to the RFID sensor antenna resonance because most applications not only have plane stretching, but also get distortion and deformation in other directions. In Fig. 8(a), the antenna was bended from $0^{\circ}$ to $90^{\circ}$ (fixed by foam holders with different angles to ensure accuracy and reproducibility), the strong coupling between the inner and outer rings makes the antenna being less sensitive to the bending, no obvious frequency shift can be observed during bending. However, the maximum read range decreases by 1 metre when the antenna is bended to $90^{\circ}$. This is because the bending distorts the antenna's radiation pattern, i.e., the gain reduces. The same conclusion can be drawn from the rolling test in Fig. 8(b) (held by paper tubes with different radii), only when the radius is smaller than 1.5 $\mathrm{cm}$, the resonance frequency starts to shift. It increases by only $15 \mathrm{MHz}$ when the rolling radius reduces to $0.7 \mathrm{~cm}$. At this point, the antenna touches itself from end to end. These experimental data verify that bending and rolling have little effects on the resonance frequency as the electric length of the antenna barely varies. 
(a)

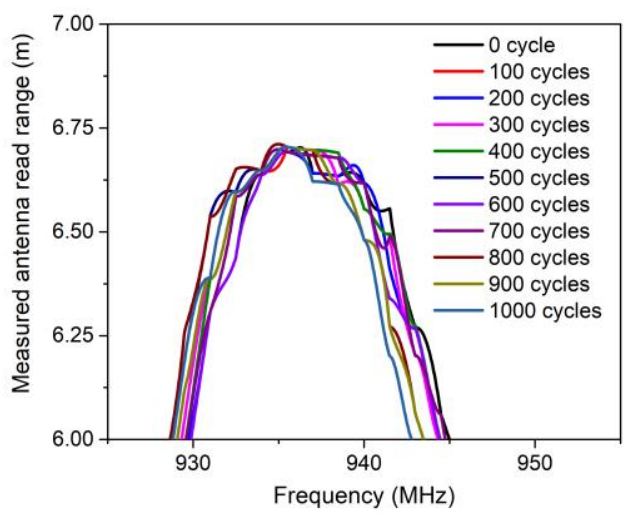

(b)

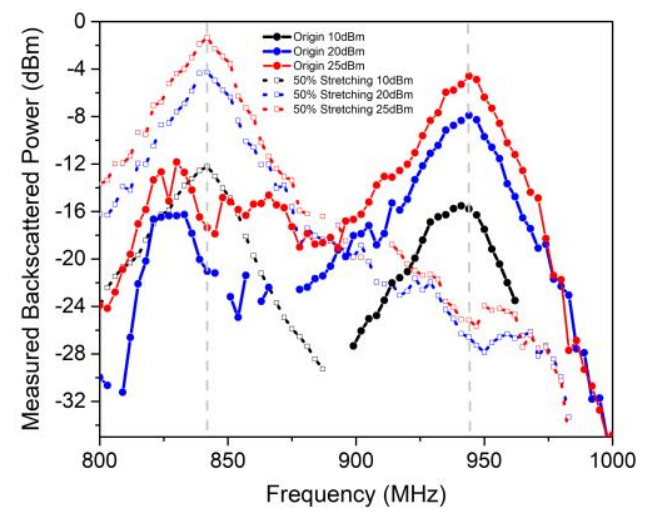

Figure 9: (a) Cyclability test of the RFID stretchable sensor. The sensor was stretched from its initial state to $200 \%$ of the original length (X direction) with 1000 cycles. (b) Demonstration of the measured backscattered power where the RFID stretchable sensor is placed in normal office environment with a fixed distance.

As the cyclability is another important criterion to evaluate stretchable sensor performance, the RFID sensor was stretched $\left(2 L_{x}\right)$ and released $\left(L_{x}\right) 1000$ cycles. Its resonance frequency was recorded every 100 cycles and plotted in Fig. 9(a). The resonance frequency is rather stable in the first 500 cycles and starts to decrease from 600 cycles. There is approximately 2 $\mathrm{MHz}$ frequency shift after 1000 cycles. Based on Eq. (7), the calculated distance error is $0.032 \mathrm{~mm}$ which confirms that the RFID sensor has an excellent cyclability.

In order to demonstrate that the proposed sensor can be used in practical environment, we moved the whole measurement set to office environment with tables, computers and people moving around. The measurement results are plotted in Fig. 9(b). In the original state, the peak value of the backscattered power varies with different radiating power whereas the resonance frequency has kept constant. After stretching the sensor of 50\%, all peak values shift from $944 \mathrm{MHz}$ to $842 \mathrm{MHz}$, indicating that the sensor works well under practical multipath interference environment.

Taking a step further to real applications, we have integrated the RFID stretchable sensor into a soft chamber which is made of silicone materials with pneumatic channels based on the structure described in [23] since it is compatible with soft lithography [24] technology. In the fabricated soft chamber, a series of pneumatic channels were embedded in an extensible
Ecoflex layer and bonded to a relatively less extensible PDMS layer (Young's Modulus of Ecoflex is $69 \mathrm{kPa}$ [13] whereas PDMS is $0.8-3 \mathrm{MPa}[25]$ ). When compressed air is injected into the chamber, the chamber inflates like balloons. The difference in strain between the Ecoflex layer and the PDMS layer causes the structure to bend.

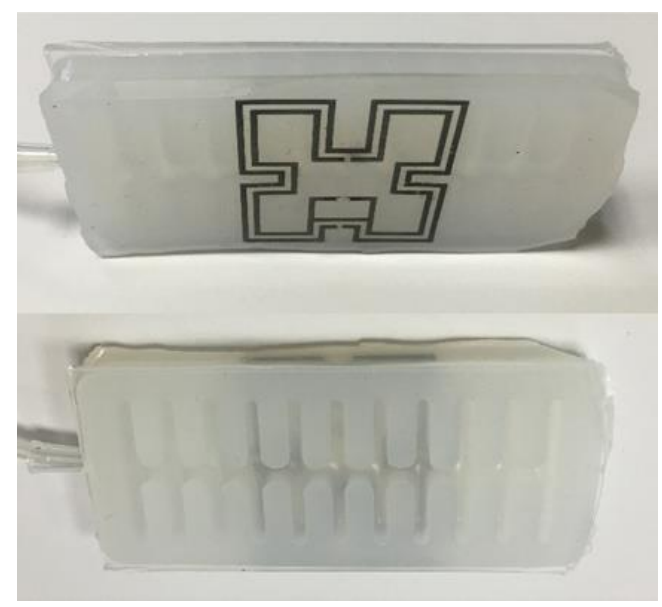

Fig. 10. Soft pneumatic actuator with RFID stretchable sensor attached on the Ecoflex side.

In Fig. 10, the fabricated actuator is flat and the RFID stretchable sensor antenna operates in its original state when air pressure is zero. Its resonance frequency is $917 \mathrm{MHz}$ (this is lower than that of the antenna shown in Fig. 4(f) because of the increment of substrate thickness). With increasing air pressure, the Ecoflex grooves start to expand. However, the expansion rate of relatively rigid PDMS layer is much smaller. The different expansion rates lead to bending action of the actuator. Under such condition, the RFID stretchable sensor was stretched at both $\mathrm{X}$ and $\mathrm{Y}$ directions and bended from $0^{\circ}$ to $90^{\circ}$. The resonance frequency with different bending angle is shown in Fig. 11. It can be seen that the average resonance frequency shift rate is $>1.4 \mathrm{MHz} /{ }^{\circ}$ at $0^{\circ} \sim 30^{\circ}$ and $>2 \mathrm{MHz} /{ }^{\circ}$ at $30^{\circ} \sim 60^{\circ}$ (exceed measurement range when bended over $60^{\circ}$ ).

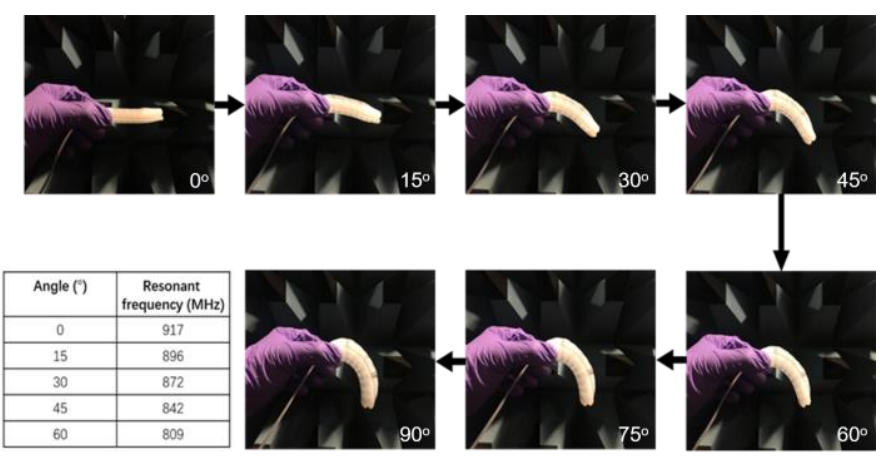

Fig. 11. Soft pneumatic actuator bending process when air pressure increasing $\left(0^{\circ}, 15^{\circ}, 30^{\circ}, 45^{\circ}, 60^{\circ}, 75^{\circ}\right.$ and $\left.90^{\circ}\right)$ and corresponded measured RFID antenna resonance frequencies. 


\section{CONCLUSION}

In this paper, we have demonstrated a metamaterial inspired soft wireless battery-free UHF RFID liquid metal stretchable sensor. The operation principle is based on the resonance frequency detection of the RFID sensor antenna, which can convert physical parameter variations to its resonance frequencies. This technique has advantages over the RSSI: it doesn't need reference antenna and has high sensitivity. First a compact SRR UHF RFID antenna which is highly sensitive to the mechanical stretch has been designed and fabricated. Secondly the performance of the RFID sensor under different stretching length, including backscattered powers, read ranges and radiation patterns, has been measured, verifying that the resonance frequency of the RFID antenna can be altered efficiently during stretching. Thirdly, bending and rolling of the antenna have been tested and discussed. Fourthly, the reliability test reveals that the sensor maintains good performance up to 1000 stretching cycles. Finally, a prototype of RFID stretching sensor enabled soft pneumatic actuator has been fabricated and demonstrated, illustrating the potential applications of the reported soft wireless battery-free UHF RFID stretchable sensor.

\section{REFERENCES}

[1] H. Stockman, "Communication by means of reflected power," Proc. IRE, vol. 36, no. 10, pp. 1196-1204, 1948.

[2] J. Landt, "The history of RFID," IEEE potentials, vol. 24, no. 4, pp. 8-11, 2005.

[3] S. Merilampi, P. Ruuskanen, T. Björninen, L. Ukkonen, and L. Sydänheimo, "Printed passive UHF RFID tags as wearable strain sensors," in Applied Sciences in Biomedical and Communication Technologies (ISABEL), 2010 3rd International Symposium on, 2010, pp. 1-5.

[4] X. Chen, L. Ukkonen, and T. Björninen, "Passive e-textile uhf rfid-based wireless strain sensors with integrated references," IEEE Sens. J., vol. 16, no. 22, pp. 7835-7836, 2016.

[5] C. Occhiuzzi, C. Paggi, and G. Marrocco, "Passive RFID strain-sensor based on meander-line antennas," IEEE Trans. Antennas Propag., vol. 59, no. 12, pp. 4836-4840, 2011.

[6] Y. Lin, C. Cooper, M. Wang, J. J. Adams, J. Genzer, and M. D. Dickey, "Handwritten, soft circuit boards and antennas using liquid metal nanoparticles," Small, vol. 11, no. 48, pp. 6397-6403, 2015.

[7] M. D. Dickey, "Emerging applications of liquid metals featuring surface oxides," ACS Appl. Mater. Interfaces, vol. 6, no. 21, pp. 18369-18379, 2014.

[8] M. Kubo et al., "Stretchable microfluidic radiofrequency antennas," Adv. Mater., vol. 22, no. 25, pp. 2749-2752, 2010.

[9] Y. Huang, Y. Wang, L. Xiao, H. Liu, W. Dong, and Z. Yin, "Microfluidic serpentine antennas with designed mechanical tunability," Lab Chip, vol. 14, no. 21, pp. 4205-4212, 2014.

[10] J. So, J. Thelen, A. Qusba, G. J. Hayes, G. Lazzi, and M. D. Dickey, "Reversibly deformable and mechanically tunable fluidic antennas," Adv. Funct. Mater., vol. 19, no. 22, pp. 3632-3637, 2009.

[11] S. H. Jeong, A. Hagman, K. Hjort, M. Jobs, J. Sundqvist, and $\mathrm{Z}$. Wu, "Liquid alloy printing of microfluidic stretchable electronics," Lab Chip, vol. 12, no. 22, pp. 4657-4664, 2012.

[12] S. H. Jeong, K. Hjort, and Z. Wu, "Tape transfer printing of a liquid metal alloy for stretchable RF electronics," Sensors, vol. 14, no. 9, pp. 16311-16321, 2014.

[13] L. Teng, K. Pan, M. P. Nemitz, R. Song, Z. Hu, and A. A. Stokes, "Soft Radio-Frequency Identification Sensors: Wireless Long-Range Strain Sensors Using Radio-Frequency Identification,”Soft Robot., 2018.

[14] J. García-García, F. Martín, J. D. Baena, R. Marques, and L. Jelinek, "On the resonances and polarizabilities of split ring resonators," J. Appl. Phys., vol. 98, no. 3, p. 33103, 2005.

[15] J. Choo, J. Ryoo, J. Hong, H. Jeon, C. Choi, and M. M. Tentzeris, "T-matching networks for the efficient matching of practical RFID tags," in Microwave Conference, 2009. EuMC 2009. European, 2009, pp. 5-8.

[16] J. B. Pendry, A. J. Holden, D. J. Robbins, and W. J. Stewart, "Magnetism from conductors and enhanced nonlinear phenomena," IEEE Trans. Microw. Theory Tech., vol. 47, no. 11, pp. 2075-2084, 1999.

[17] R. Marqués, F. Mesa, J. Martel, and F. Medina, "Comparative analysis of edge-and broadside-coupled split ring resonators for metamaterial design-theory and experiments," IEEE Trans. Antennas Propag., vol. 51, no. 10, pp. 2572-2581, 2003.

[18] B. Sauviac, C. R. Simovski, and S. A. Tretyakov, "Double split-ring resonators: Analytical modeling and numerical simulations," Electromagnetics, vol. 24, no. 5, pp. 317-338, 2004.

[19] R. Marqués, F. Medina, and R. Rafii-El-Idrissi, "Role of bianisotropy in negative permeability and left-handed metamaterials," Phys. Rev. B, vol. 65, no. 14, p. 144440, 2002.

[20] T. Liu, P. Sen, and C.-J. Kim, "Characterization of nontoxic liquid-metal alloy galinstan for applications in microdevices," J. Microelectromechanical Syst., vol. 21, no. 2, pp. 443-450, 2012.

[21] S. Zuffanelli, G. Zamora, P. Aguilà, F. Paredes, F. Martín, and J. Bonache, "On the radiation properties of split-ring resonators (SRRs) at the second resonance," IEEE Trans. Microw. Theory Tech., vol. 63, no. 7, pp. 2133-2141, 2015.

[22] J.-B. Chossat, Y.-L. Park, R. J. Wood, and V. Duchaine, "A soft strain sensor based on ionic and metal liquids," IEEE Sens. J., vol. 13, no. 9, pp. 3405-3414, 2013.

[23] F. Ilievski, A. D. Mazzeo, R. F. Shepherd, X. Chen, and G. M. Whitesides, "Soft robotics for chemists," Angew. Chemie, vol. 123, no. 8, pp. 1930-1935, 2011.

[24] D. Qin, Y. Xia, and G. M. Whitesides, "Soft lithography for micro-and nanoscale patterning," Nat. Protoc., vol. 5, no. 3, p. 491, 2010.

[25] Y. Fouillet et al., "Stretchable Material for Microfluidic Applications," in Multidisciplinary 
Digital Publishing Institute Proceedings, 2017, vol. 1, no. 4, p. 501.

[26] CST (Computer Simulation Technology). https://www.cst.com/ 